\title{
Bursts of low-energy electron-positron pairs in TeV-range collider physics
}

\author{
Francesco Giacosa ${ }^{\dagger}$ and Ralf Hofmann* \\ $\dagger$ Institut für Theoretische Physik \\ Universität Frankfurt \\ Johann Wolfgang Goethe - Universität \\ Max von Laue-Str. 1 \\ 60438 Frankfurt, Germany \\ * Institut für Theoretische Physik \\ Universität Karlsruhe (TH) \\ Kaiserstr. 12 \\ 76131 Karlsruhe, Germany
}

\begin{abstract}
In this Letter we investigate the possible emission of low-energy electron neutrinos and electron-positron pairs of anomalously large multiplicity in close-to-central $p p$ collisions at LHC. The scenario is based on confining $\mathrm{SU}(2)$ Yang-Mills dynamics of Hagedorn temperature $\sim m_{e}=511 \mathrm{keV}$ being responsible for the emergence of the lightest lepton family and the weak interactions of the Standard Model. Although cut off by LHC's detectors these electronspositron bursts would be seen indirectly by a large defect energy and thus an anomalously strong decrease of events with interesting high-energy secondaries for increasing $\sqrt{s}$. This is because the formation of superconducting (preconfining) $\mathrm{SU}(2)$ hot-spots 'steals' a large fraction of $\sqrt{s}$ subsequently transferring it to a thermal spectrum of electron neutrinos, electrons, and positrons liberated through evaporation. We thus propose the detection of electrons and positrons of kinetic energy $\sim m_{e}$ and photons of energy $\sim 2 m_{e}$.
\end{abstract}


Introduction. The experimental program at LHC will start soon. In this note we wish to point out that large multiplicities in $e^{+} e^{-}$production and a spectrum of gamma rays strongly peaked at $\sim 1 \mathrm{MeV}$ are expected to occur based on the scenario that the emergence and the weak interactions of electrons, positrons, and their neutrinos are due to a pure $\mathrm{SU}(2)$ Yang-Mills theory of scale $\Lambda_{e} \sim m_{e}=$ $0.511 \mathrm{MeV}$ [1, 2, 3, 4, 5. Being highly unconventional, this framework needs further elaboration and tests to be viable. Nevertheless, we feel that some consequences of this approach to electroweak physics could be of relevance in interpreting certain experimental signatures at LHC. It is suspected that an unexpectedly low rate of events with an energy-momentum transfer comparable to the center-of-mass energy of colliding protons occurs.

In 11, 3, 4, we have explained how the confining phase of an SU(2) Yang-Mills theory of scale $\sim 0.5 \mathrm{MeV}$ provides for the emergence of stable and instable fermionic solitons (center-vortex loops) where the three lightest excitations are interpreted as the (Majorana) electron-neutrino and the electron/positron, respectively. These nonlocal excitations [5] are liberated when cooling the Yang-Mills system below the Hagedorn temperature $T_{\mathrm{H}} \sim m_{e}=0.511 \mathrm{MeV}$. Shortly above $T_{\mathrm{H}}$ the theory is preconfining [1] with the dual massive gauge mode decoupling at $T_{\mathrm{H}}$. When disregarding the mixing with $\mathrm{U}(1)_{Y}$ this neutral vector boson is interpreted as the $Z_{0}$ resonance. Above $T_{c}=1.14 T_{\mathrm{H}}$ there is a deconfining phase, and two massive and oppositely charged vector bosons decouple at $T_{c}$. These modes are interpreted as the $W^{ \pm}$resonances. In this scenario the existence of the mass hierarchy $m_{Z_{0}} / m_{e} \sim$ $m_{W^{ \pm}} / m_{e} \sim m_{e} / m_{\nu_{e}} \sim 10^{5}$ is not explained by the large vacuum expectation of an elementary and fundamentally $\mathrm{SU}(2)$ charged scalar field - the Higgs field of the Standard Model, see for instance the review of Ref. [6] and Refs. therein - but is related to the large values of effective gauge couplings at their respective phase boundaries. Notice that the dynamical (self-induced) gauge-symmetry breaking, compare also with Refs. [7], is a consequence of the nonperturbative sector of the pure $\mathrm{SU}(2)$ Yang-Mills theory which, after a suitable spatial coarse-graining, manifests itself in terms of effective and inert (non-fluctuating) scalar fields.

In this note we compute decay signatures of preconfining (superconducting) hotspots of Bohr-radius size as they may be generated in head-on proton collisions at several $\mathrm{TeV}$ center-of-mass energy. These signatures include temporal event shapes, evaporation times, and lepton-emission multiplicities. In the case that the $p p$-collision products at LHC are also viewed at energies comparable to $m_{e}$ these signatures may prove to be relevant. Indirectly, we suspect an unexpectedly rare detection of events with large four-momentum transfer because the above-mentioned hot-spots 'steal' out of the collision zone considerable fractions of the original centerof-mass energy to redistribute this energy into large numbers of slow electrons and positrons (kinetic energy $\sim m_{e}$ ). This is not unlike the greenhouse effect where a high-frequency photon after traversing the glass-wall from outside is transformed into a large number of low-frequency photons which can not escape the house anymore. The analysis performed in the present work is based on simple geometric 
considerations and on energy-conservation. We work in natural units where the speed of light in vacuum and Boltzmann's constant are set equal to unity.

Size, lifetime, and decay products of superconducting hot-spots. For $T \sim T_{\mathrm{H}}$ the energy density $\rho_{\mathrm{H}}$ in the preconfining phase is given as $\rho_{\mathrm{H}}=4 \pi \Lambda^{3} T_{\mathrm{H}}$ where $\Lambda \sim m_{e}$ [1, 2] is the Yang-Mills scale of the $\mathrm{SU}(2)$ gauge theory. Notice that $\rho_{\mathrm{H}}$ solely is due to the thermal ground state in that phase because the massive, dual gauge mode decouples at $T_{\mathrm{H}}$. To parameterize an uncertainty in the relation between $\Lambda$ and $m_{e}$ we introduce the dimensionless parameter $y$ as $\Lambda=y m_{e}$. As a consequence, $T_{\mathrm{H}}$ can be expressed as $T_{\mathrm{H}}=\frac{11.24}{2 \pi} y m_{e}$ [2], and we have $\rho_{\mathrm{H}}=22.48 y^{4} m_{e}^{4}$. Moreover, we denote by $\delta$ the fraction of the total center-of-mass energy $\sqrt{s} \sim 14 \mathrm{TeV}$ used up by the formation of a spherical $\mathrm{SU}(2)_{e}$ hot-spot (preconfining phase) of initial radius $R_{0}$. Assuming the hot-spot to be homogeneously thermalized 1 at $T_{\mathrm{H}}$, the initial radius $R_{0}$ calculates as

$$
\delta \sqrt{s}=\rho_{\mathrm{H}} \frac{4}{3} \pi R_{0}^{3} \Rightarrow R_{0}=\left(\frac{3}{4 \pi} \frac{\delta \sqrt{s}}{\rho_{\mathrm{H}}}\right)^{1 / 3} .
$$

The left panel in Fig. 1 depicts the dependence of $R_{0}$ (in units of the Bohr radius $a_{\mathrm{Bohr}}=5.29 \times 10^{-11} \mathrm{~m}$ ) on $\delta$ for $y=1 / 2$ and $y=1$, corresponding to $T_{\mathrm{H}}=0.89 \mathrm{~m}_{e}$ and $T_{\mathrm{H}}=1.79 m_{e}$, respectively. Notice that on the scale of the proton radius the initial hot-spot radius $R_{0}$ is gigantic for $\delta \sim O(1)$.

Once it has formed, the hot-spot starts to decay by the emission of single and self-intersecting center vortex loops from its surface. The latter defines the spatial location where the Hagedorn transition towards confining dynamics takes place (traversed from inside to outside). Asymptotically far away from the hot-spot's surface only (quasi)stable solitons, see also [5], survive. These mainly are $\nu_{e}$ 's and $e^{+}-e^{-}$pairs roughly being thermalized at $T_{\mathrm{H}}$. Close to the surface the plasma is highly turbulent [3, 4] because of the production and decay of unstable fermions with masses $m_{n} \sim n \cdot m_{e}$ with $n=2,3, \ldots$ into $\nu_{e}$ and $e^{+}-e^{-}$pairs, and, occasionally, into $1 \mathrm{MeV}-\gamma$ 's.

For the hypothetical situation that all fermions are thermalized at a temperature $T$ the energy density $\varepsilon_{n}$ belonging to fermions of mass $m_{n}$ is given as

$$
\varepsilon_{n}(T) \equiv \int_{0}^{\infty} d p u_{n}(p, T) \equiv \int_{0}^{\infty} d p p^{2} \frac{M_{n}}{2 \pi^{2}} \frac{\sqrt{p^{2}+m_{n}^{2}}}{e^{\beta \sqrt{p^{2}+m_{n}^{2}}}+1}
$$

where $M_{0}=2$ (Majorana neutrinos, only spin degeneracy [8]), $M_{1}=2 \cdot 2=4$ for $e^{+}$ and $e^{-}$(spin and charge degeneracy), and $\beta \equiv 1 / T$. To evaluate the emissive power

\footnotetext{
${ }^{1}$ In reality, the radius of the hot-spot will undergo complicated oscillations since at $T_{\mathrm{H}}$ the pressure $p=p_{\mathrm{H}}=-\rho_{\mathrm{H}}$ is maximally negative, thus shrinking the hot-spot, while the pressure $p$ becomes positive just above the deconfining temperature $T_{c}=1.14 T_{\mathrm{H}}$, thus expanding the hotspot. This situation should be describable by ideal hydrodynamics. However, since the spread of the associated temperature is small, we neglect this effect in the following and assume homogeneous thermalization at $T_{\mathrm{H}}$.
} 

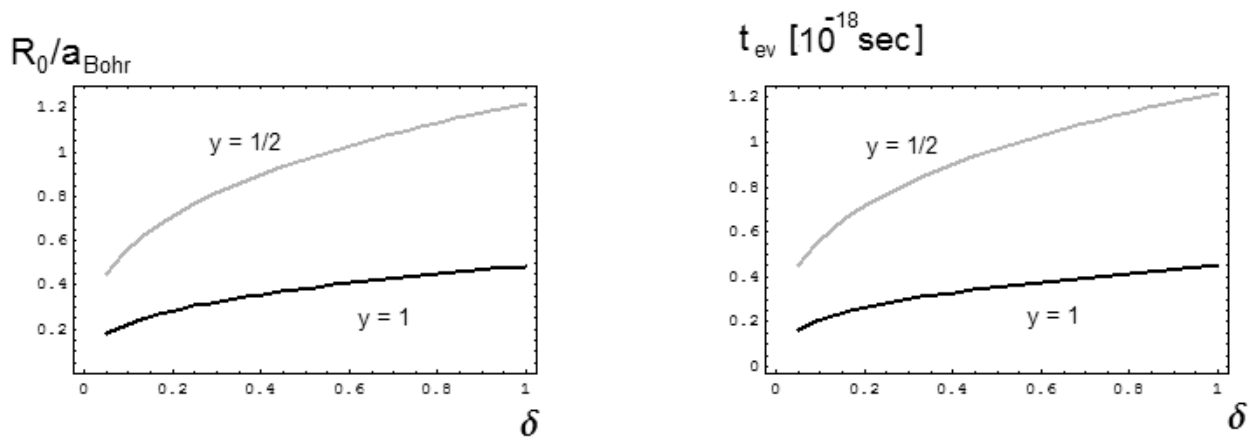

Figure 1: Left panel: Initial hot-spot radius $R_{0}$ in units of the Bohr radius as a function of $\delta$ for $y=1 / 2$ (gray curve) and $y=1$ (black curve). Right panel: Evaporation time $t_{e v}$ in units of $10^{-18} \mathrm{sec}$ as a function of $\delta$ for $y=1 / 2$ (gray curve) and $y=1$ (black curve).

$J_{n}(T)$ (energy emitted per unit time and unit surface) of fermions with mass $m_{n}$ we multiply the spectral density $u_{n}(p, T)$ with $v_{n} / 4\left[9\right.$. Here the velocity $v_{n}=v_{n}(p)$ is given as $v_{n}=p / \sqrt{p^{2}+m_{n}^{2}}$. Thus, we have

$$
J_{n}(T)=\int_{0}^{\infty} d p \frac{v_{n}}{4} u_{n}(p, T)=\int_{0}^{\infty} d p \frac{M_{n}}{8 \pi^{2}} \frac{p^{3}}{e^{\beta \sqrt{p^{2}+m_{n}^{2}}}+1} .
$$

The total emissive power is then given as $J(T)=\sum_{n=0}^{\infty} J_{n}(T)$. A similar series was studied in [3] demonstrating its asymptotic nature. The latter, in turn, was shown to be responsible for the emergence of non-equilibrium behavior (imaginary part in the pressure) by analytically continuing the Borel resummed series back to physical 'temperatures' [4. Since we are only interested in what is observed far from the emitting surface we safely may assume a thermal freeze-out at $T_{\mathrm{H}}$ of the (quasi)stable excitations $\nu_{e}$ and $e^{+}-e^{-}$. Thus $J\left(T_{\mathrm{H}}\right)=J_{0}\left(T_{\mathrm{H}}\right)+J_{1}\left(T_{\mathrm{H}}\right)$.

Let us now turn to the time-evolution of the hot-spot radius $R=R(t)$. To do this, recall that we assume the hot-spot to be homogeneously thermalized at $T_{\mathrm{H}}$. Seen from far away, the hot-spot evaporates at a temperature $T_{\mathrm{H}}$ into $\nu_{e}$ and $e^{+}-e^{-}$ where $R\left(t_{0}=0\right)=R_{0}$ as evaluated above. By energy conservation, the differential equation describing this evaporation reads as

$$
\frac{d E}{d t}=\frac{d}{d t}\left(\frac{4}{3} \pi R^{3} \rho_{\mathrm{H}}\right)=-J\left(T_{\mathrm{H}}\right) \cdot 4 \pi R^{2}
$$

where $E=\frac{4}{3} \pi R^{3} \rho_{\mathrm{H}}$ denotes the total energy contained in the hot-spot at time $t$. The solution is easily obtained:

$$
R(t)=R_{0}-\frac{J\left(T_{\mathrm{H}}\right)}{\rho_{\mathrm{H}}} t
$$


Thus, the time $t_{e v}$ needed for complete evaporation of the hot-spot reads

$$
t_{e v}=\frac{\rho_{\mathrm{H}}}{J\left(T_{\mathrm{H}}\right)} R_{0}
$$

In the right panel of Fig. 1 the quantity $t_{e v}$ is plotted as a function of $\delta$ for $y=1 / 2$ and $y=1$ indicating that $t_{e v}$ is of order $10^{-18} \mathrm{sec}$. Thus an initially large hot-spot (Bohr-radius size) exhibits a decay time typically observed in electromagnetic decays of hadrons!

Let us now evaluate the number of fermions emitted. We denote by $\eta_{n}$ the number of fermions of mass $m_{n}$ emitted per unit time and surface, and we have

$$
\eta_{n}(T)=\int_{0}^{\infty} d p \frac{v_{n}}{4} \frac{u_{n}(p, T)}{\sqrt{p^{2}+m_{n}^{2}}}=\int_{0}^{\infty} d p \frac{M_{n}}{8 \pi^{2} \sqrt{p^{2}+m_{n}^{2}}} \frac{p^{3}}{e^{\beta \sqrt{p^{2}+m_{n}^{2}}}+1} .
$$

As before, we may restrict to the cases $n=0$ and $n=1$ after thermal freeze-out. The total numbers $N_{\nu_{e}}$ and $N_{e^{+-} e^{-}}$of neutrinos and $e^{+}, e^{-}$emitted are given as

$$
\begin{gathered}
N_{\nu_{e}}=\int_{0}^{t_{e v}} d t n_{\nu_{e}}(t), \quad n_{\nu_{e}}(t) \equiv 4 \pi \eta_{0}\left(T_{\mathrm{H}}\right) R(t)^{2}, \\
N_{e^{+}-e^{-}}=\int_{0}^{t_{e v}} d t n_{e^{+}-e^{-}}(t), \quad n_{e^{+-e^{-}}}(t) \equiv 4 \pi \eta_{1}\left(T_{\mathrm{H}}\right) R(t)^{2} .
\end{gathered}
$$

The quantities $\eta_{0}\left(T_{\mathrm{H}}\right)$ and $\eta_{1}\left(T_{\mathrm{H}}\right)$ are constant implying that $N_{\nu_{e}}=c \eta_{0}\left(T_{\mathrm{H}}\right)$ and $N_{e^{+-e^{-}}}=c \eta_{1}\left(T_{\mathrm{H}}\right)$ with the universal constant $c$ defined as $c \equiv 4 \pi \int_{0}^{t_{e v}} d t R(t)^{2}$. In the left panel of Fig. 2 the numbers $N_{\nu_{e}}$ and $N_{e^{+-} e^{-}}$are shown as functions of $\delta$ for $y=1 / 2$. Notice that for $\delta=O(1)$ a very large number of fermions is emitted and that the dependence on $\delta$ is rather weak. Finally, in the right panel of Fig. 2 the emission rates $n_{\nu_{e}}(t)$ and $n_{e^{+-} e^{-}}(t)$ are plotted for $y=\delta=1 / 2$. Notice that the burst is particularly strong just after formation with rapidly decreasing rates thereafter. The situation does not change qualitatively when varying $y$ within $0.1 \leq y \leq 1$.

Discussion and Conclusion. In this Letter we have calculated the initial radius, the lifetime, the emission rates and total numbers of neutrinos and electrons/positrons emitted by superconducting hot-spots generated at LHC energies. These hot-spots represent the preconfining thermal ground state in pure SU(2) YangMills theory with the latter assumed to be responsible for the emergence of the lightest lepton family and the weak interactions [1, 2]. At LHC this mechanism may serve as an energy-shifter in the sense that the creation of preconfining hot-spots 'steals' a large fraction of the initial center-of-mass energy $\sqrt{s}$ from the $p p$ collision zone subsequently pumping it into the creation of a large number of thermal electrons, positrons and neutrinos at a temperature comparable to $m_{e}=0.511 \mathrm{MeV}$. If this mechanism would become more effective with increasing $\sqrt{s}$, then this would imply that the number of interesting high-energy events quickly dies off. In addition, the superconductivity of the hot-spot 'stuff' creates magnetic fields that locally compete 

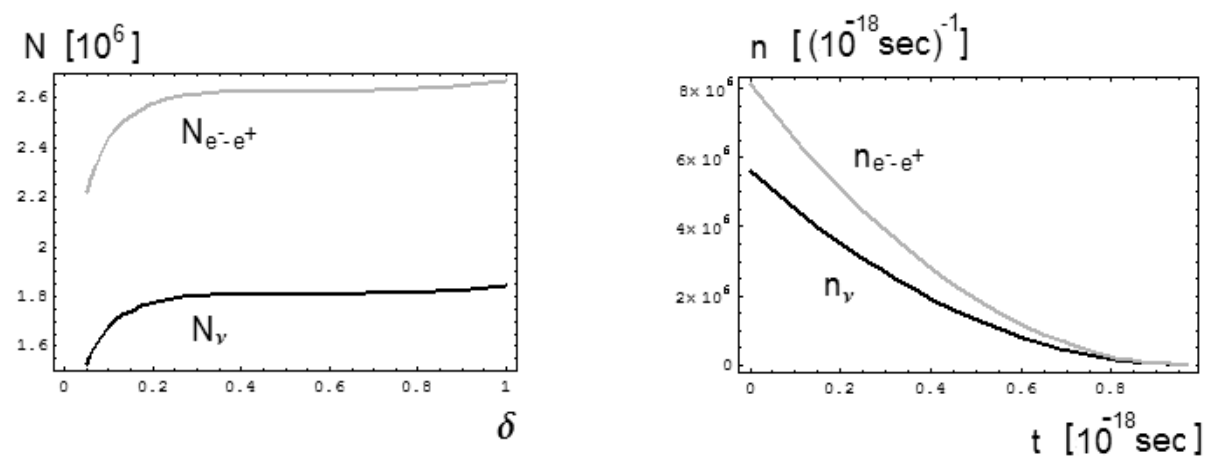

Figure 2: Left panel: number $N_{\nu_{e}}$ of neutrinos emitted (black curve) and number $N_{e^{-}-e^{+}}$electrons and positrons emitted (gray curve) as functions of $\delta$ for $y=1 / 2$. Right panel: Emission rates for neutrinos (black) and for electrons/positrons (grey) for $y=1 / 2$ and $\delta=1 / 2$.

with external magnetic fields and thus defocus the beam in the collision zone. By the annihilation of electron-positron pairs we also expect a gamma spectrum which is strongly peaked at an energy of about $1 \mathrm{MeV}$.

Notice that there are two major differences between our scenario and black-hole evaporation [10]: (i) Evaporation of black hole is induced by the universal coupling of gravity to matter, and thus all kinds of sufficiently stable particles are expected to appear as decay products while we only expect a large number of neutrinos, electrons and positrons plus a spectrum of photons peaked at $\sim 1 \mathrm{MeV}$. (ii) The rate of particle emission due to our preconfining hot spots is large in an initial stage just after formation and decreases thereafter. This is opposite to what is expected during black-hole evaporation.

Finally, let us point out that the confining phase of an SU(2) Yang-Mills theory of scale $\sim m_{\mu} \sim 200 m_{e} \sim 100 \mathrm{MeV}$ would provide for the emergence of the second lepton family. The triplet $Z_{0}^{\prime}$ and $W_{ \pm}^{\prime}$ of heavy vector bosons of that theory would mediate very weak interactions. Assuming the same decoupling values $\sim 10^{5}$ of the effective gauge couplings as experimentally inferred for the $\mathrm{SU}(2)$ theory discussed above, the masses within this additional triplet would be of the order of $10^{5} m_{\mu} \sim$ $10 \mathrm{TeV}$ which is just within the reach of LHC.

\section{References}

[1] R. Hofmann, Int. J. Mod. Phys. A20, 4123 (2005), Erratum-ibid. A21, 6515 (2006).

[2] F. Giacosa and R. Hofmann, Eur. Phys. J C50, 635 (2007). 
[3] F. Giacosa, R. Hofmann, and M. Schwarz, Mod. Phys. Lett. A21, 2709.

[4] R. Hofmann, arXiv:0710.0962 [hep-th].

[5] J. Moosmann and R. Hofmann, arXiv:0804.3527 [hep-th].

[6] A. Djouadi, Phys. Rept. 457 (2008) 1.

[7] G. 't Hooft, Nucl. Phys. B 138 (1978) 1; L. D. Faddeev and A. J. Niemi, Phys. Rev. Lett. 82 (1999) 1624.

[8] H. V. Klapdor-Kleingrothaus, I. V. Krivosheina, A. Dietz and O. Chkvorets, Phys. Lett. B 586 (2004) 198; H. V. Klapdor-Kleingrothaus, Int. J. Mod. Phys. D 13 (2004) 2107.

[9] See, for instance, W. Greiner, L. Neise, H. Stöcker, Thermodynamik und Statistische Mechanik Verlag Harri Deutsch, Frankfurt, 1. Auflage (1987); http://en.wikipedia.org/wiki/Stefan-Boltzmann_law.

[10] D. N. Page, New J. Phys. 7 (2005) 203; D. M. Gingrich, JHEP 0711 (2007) 064. 